\title{
Cerebrospinal Fluid Catecholamine Levels as Predictors of Outcome in Subarachnoid Hemorrhage
}

\author{
Michael Moussouttas ${ }^{a}$ Thanh T. Huynh ${ }^{c}$ John Khoury ${ }^{b}$ Edwin W. Lai ${ }^{c}$ \\ Keith Dombrowski ${ }^{b}$ Scott Pello ${ }^{b}$ Karel Pacak ${ }^{c}$ \\ ${ }^{a}$ Cerebrovascular and Neurocritical Care Division, and ${ }^{b}$ Department of Neurology, Thomas Jefferson \\ Medical Center, Philadelphia, Pa., and 'Section on Medical Neuroendocrinology, Reproductive and Adult \\ Endocrinology Program, National Institutes of Health, Bethesda, Md., USA
}

\section{Key Words}

Subarachnoid hemorrhage $\cdot$ Sympathetic nervous system $\cdot$ Catecholamines $\cdot$ Cerebrospinal fluid $\cdot$ Prognosis $\cdot$ Outcome

\begin{abstract}
Objective: Subarachnoid hemorrhage (SAH) is associated with marked sympathetic activation at the time of ictus. The purpose of this study is to determine whether early central catecholamine levels measured from cerebrospinal fluid (CSF) relate to outcome in patients with SAH. Methods: Observational study of consecutive SAH grade 3-5 patients who underwent ventriculostomy placement, but did not undergo open craniotomy for aneurysm obliteration. CSF samples were obtained during the first $48 \mathrm{~h}$ following symptom onset and assayed for catecholamine levels. Statistical analyses were performed to determine whether the levels predicted mortality by day 15 or mortality/disability by day 30 . Results: For the 102 patients included, mean age was 58, and $73 \%$ were female $-21 \%$ experienced day- 15 mortality, and $32 \%$ experienced mortality/disability by day 30 . Early mortality was related to Hunt-Hess $(H / H)$ grade $(p<0.001)$, neu-
\end{abstract}

rogenic cardiomyopathy $(\mathrm{NC})(\mathrm{p}=0.003)$, cerebral infarction $(p=0.001)$, elevated intracranial pressure (ICP) $(p=0.029)$, epinephrine (EPI) level $(p=0.002)$ and norepinephrine/3,4dihydroxyphenylglycol (NE/DHPG) ratio $(p=0.003)$. Mortality/disability was related to $\mathrm{H} / \mathrm{H}$ grade $(p<0.001), \mathrm{NC}(\mathrm{p}=$ $0.018)$, infarction $(p<0.001)$, elevated ICP $(p=0.002)$, EPI ( $p=$ $0.004)$ and NE/DHPG $(p=0.014)$. Logistic regression identified age [OR $1.09(95 \% \mathrm{Cl} 1.01-1.17)], \mathrm{H} / \mathrm{H}$ grade [9.52 (1.1977)], infarction [10.87 (1.22-100)], ICP elevation [32.26 (2500)], EPI [1.06 (1.01-1.10)], and (inversely) DHPG [0.99 (0.991.00)] as independent predictors of early mortality. For mortality/disability, H/H grade [OR 21.74 (95\% Cl 5.62-83)], ICP elevation [18.52 (1.93-166)], and EPI [1.05 (1.02-1.09)] emerged as independent predictors. Proportional-hazards analysis revealed age [HR $1.041(95 \% \mathrm{Cl} 1.003-1.08)], \mathrm{H} / \mathrm{H}$ grade [6.9 (1.54-31.25)], NC [4.31 (1.5-12.35)], and EPI [1.032 (1.009-1.054)] independently predicted early mortality. Conclusions: CSF catecholamine levels are elevated in SAH patients who experience early mortality or disability. EPI may potentially serve as useful index of outcome in this population of patients with SAH.

Copyright $\odot 2012$ S. Karger AG, Basel

\section{KARGER}

Fax +4161306 1234 E-Mail karger@karger.ch www.karger.com (c) 2012 S. Karger AG, Basel

1015-9770/12/0332-0173\$38.00/0

Accessible online at:

www.karger.com/ced
Michael Moussouttas, MD

900 Walnut Street, Suite 200

Philadelphia, PA 19107 (USA)

Tel. +1 2152790722

E-Mail arista1@pol.net 


\section{Introduction}

Biological molecular markers are being increasingly tested and employed in the diagnostic and prognostic evaluation of patients with cerebrovascular diseases $[1,2]$. Biomarkers have been evaluated and utilized in the diagnosis of cerebral ischemic and hemorrhagic events [3], in predicting hemorrhagic complications from fibrinolytic therapy, in predicting an aggressive clinical course for middle cerebral artery infarctions, and in determining outcome $[1,2]$.

Early prognostication for patients with intracranial hemorrhage may provide crucial information regarding treatment options and family decisions. For patients with intracerebral hemorrhage (ICH) grading scales incorporating proven demographic, clinical and radiological predictors of mortality have been developed and validated for determining outcome $[4,5]$. By contrast, no analogous validated multifactorial grading scale exists for reliably predicting outcome among patients with subarachnoid hemorrhage (SAH).

SAH is associated with sudden profound sympathetic activation [6], which may promote systemic inflammation [7], precipitate thrombotic processes [8], and cause cardiopulmonary dysfunction [9], all of which contribute to adverse outcomes in cerebrovascular disease $[10,11]$. Inflammatory, thrombotic and cardiovascular biomarkers have previously been investigated as potential predictors of outcome in patients with SAH. The purpose of this study is to determine whether measures of acute central catecholamine activity may also serve as markers and predictors for early mortality and disability in patients with $\mathrm{SAH}$.

\section{Methods}

Study Overview, Data Collection, Primary Endpoints

This investigation is an observational study of consecutive patients with primary nonrecurrent SAH. Demographic, clinical, radiologic, and laboratory data were abstracted from the medical record or obtained from family interviews. During the first $48 \mathrm{~h}$ following the ictus, all enrolled patients underwent cerebral spinal fluid (CSF) sampling for analysis. The primary endpoints were early mortality occurring by day 15 , and poor outcome defined as mortality or disability with a Glasgow Coma Scale score of $\leq 10$ by day 30 [12]. Glasgow Coma Scale assessments are routinely performed every $2 \mathrm{~h}$ on all service patients.

\section{Inclusion/Exclusion Criteria}

Inclusion criteria included age $\geq 18$ years; clinical Hunt-Hess $(\mathrm{H} / \mathrm{H})$ grade 3-5 upon presentation; insertion of a functioning ventriculostomy drain; endovascular aneurysmal treatment (open surgery not performed); collection of CSF within $48 \mathrm{~h}$ of onset, and informed consent. Exclusion criteria included preexisting severe cardiopulmonary disease; SAH due to trauma, arteriovenous malformation, $\mathrm{ICH}$, arterial dissection or unusual/ atypical causes; recent or distantly recurrent SAH; and prior placement of a ventriculoperitoneal (or ventriculopleural) shunt.

\section{CSF Collection and Catecholamine Assay}

All CSF samples were obtained by ventriculostomy drainage, following the waste of any CSF already present in the tubing. Using standard tubing systems with a drainage port of fixed distance from the ventriculostomy catheter, aspiration of $1 \mathrm{ml}$ consistently removes all intraluminal CSF and allows for subsequent sample acquisition directly from the ventricular system. CSF samples $(1 \mathrm{ml})$ were collected in a glass red-top-tube and immediately placed in a deep freeze at $-80^{\circ} \mathrm{C}$. After defrosting and brief centrifugation, $0.3-\mathrm{ml}$ aliquot samples of supernatant were assayed by high performance liquid chromatography for several catecholamine compound and metabolite levels - epinephrine (EPI), norepinephrine (NE), 3,4-dihydroxyphenylglycol (DHPG), 3,4-dihydroxyphenylalanine (DOPA), dopamine (DA), 3,4-dihydroxyphenyl acetic acid (DOPAC) - using a previously described technique [13]

\section{Data Grouping}

Due to the low number of $\mathrm{H} / \mathrm{H}$ grade 5 patients, grades 4 and 5 were grouped and the clinical grade variable was dichotomized between grade 3 and grade $4 / 5$ for all calculations. Admission CT scans were scored for quantification of hemorrhage using the technique developed by Frontera et al., which provides the most linear correlation between radiographic severity and risk for complications and adverse outcomes [14]. Due to a disproportion in the distribution of CT scores, scores 1 and 2 and scores 3 and 4 were combined, respectively, and the radiological score variable was dichotomized between scores $1 / 2$ and $3 / 4$ for all calculations.

\section{SAH Complications}

Neurogenic cardiomyopathy (NC) was determined using transthoracic echocardiography performed within 2 days of onset, and was defined by characteristic myocardial dysfunction not conforming to a single vascular territory or attributable to any alternative process, demonstrating partial or complete resolution on repeat transthoracic echocardiography several days later [15]. Vasospasm was identified using transcranial ultrasound, which is routinely performed twice daily in all patients, and defined according to the following mean velocities $\left(\mathrm{V}_{\mathrm{m}}\right)$ and velocity ratios $\left(V_{\mathrm{r}}\right)$ - middle cerebral artery $\mathrm{V}_{\mathrm{m}}>120 \mathrm{~cm} / \mathrm{s}$ plus middle cerebral artery/internal cerebral artery $\mathrm{V}_{\mathrm{r}}>3$, and anterior cerebral artery $\mathrm{V}_{\mathrm{m}}>100[16,17]$. Infarction was defined as any acute arterial territorial infarct developing at any time during hospitalization as determined by cerebral CT imaging. Continuous ICP monitoring was performed via the inserted ventriculostomy catheter, which was maintained closed and opened only for reasons of ICP reduction or acquisition of laboratory samples. ICP elevation was defined as pressures persistently above $20 \mathrm{~mm} \mathrm{Hg}$ for at least $10 \mathrm{~min}$, not remedied by simple maneuvers such as head elevation or ventriculostomy drainage, and requiring aggressive intervention for normalization (ex hyperosmolar agents, sedation or hypothermia), at any time during hospitalization. 
Statistical Analyses

For comparisons of categorical variables, the Fisher's exact test or $\chi^{2}$ tests were used, and for comparisons of continuous variables the independent (two-tailed) $t$ test and nonparametric rank sum tests were used. Catecholamine variables were interrogated for collinearity using appropriate statistical diagnostic tests. Variables demonstrating a possible association in the univariate analyses $(\mathrm{p}<0.1)$ were entered into logistic regression models using the stepwise backward elimination method. All variables revealing a possible association $(\mathrm{p}<0.1)$ with mortality were entered into Cox proportional (hazards) regression models using stepwise backward elimination. For all analyses, a p value $<0.05$ was considered statistically meaningful. Outcome measures were presented according to assay level or ratio quartiles, and corresponding receiver operating characteristic (ROC) curves, and proportional-hazards survival curves, were constructed.

\section{Study Location, Approval and Ethics}

Eligible and enrolled patients were all admitted to the neurological intensive care unit at the Jefferson Hospital of Neurosciences. The present study was approved by the Committee on $\mathrm{Hu}$ man Research at Thomas Jefferson University Medical Center, and informed consent was obtained from all patients or family members for the acquisition of CSF samples. Laboratory researchers assaying CSF catecholamine levels were unfamiliar with the demographic, clinical or radiologic details of the patients, or with the occurrence of any of the primary-outcome measures.

\section{Results}

To obtain the study group, a total of 353 patients were screened. From these, 161 were excluded due to low SAH grade $(\mathrm{H} / \mathrm{H} 1 / 2)$ or lack of need for ventriculostomy $(\mathrm{H} / \mathrm{H}$ 3 without hydrocephalus), 35 were excluded due to moribund condition and/or discontinuation of support, 14 presented more than $48 \mathrm{~h}$ after onset, 4 had atypical reasons for SAH (2 endocarditis and 2 dissections), 4 were screened too late, 3 had preexisting severe cardiac problems ( 2 cardiomyopathies and 1 severe pulmonary hypertension), 2 refused enrollment, 1 was a previously treated rehemorrhage, and 1 had a preplaced ventriculoperitoneal shunt. Of the remaining patients, 25 had undergone surgical clipping of aneurysm, and 1 had insufficient CSF sample for assay.

For the 102 patients included, mean age was 58, and $73 \%$ were female. Baseline features of the study group are presented in table 1. Details regarding neurological condition, day of mortality or final clinical assessment are provided in table 2 . Of the entire group, $21 \%$ experienced early mortality, and 32\% experienced early mortality or disability. In the univariate analysis, early mortality was related to $\mathrm{H} / \mathrm{H}$ grade $(\mathrm{p}<0.001), \mathrm{NC}(\mathrm{p}=0.003)$, infarction $(\mathrm{p}=0.001)$, and elevated ICP $(\mathrm{p}=0.029)$. Early mor-

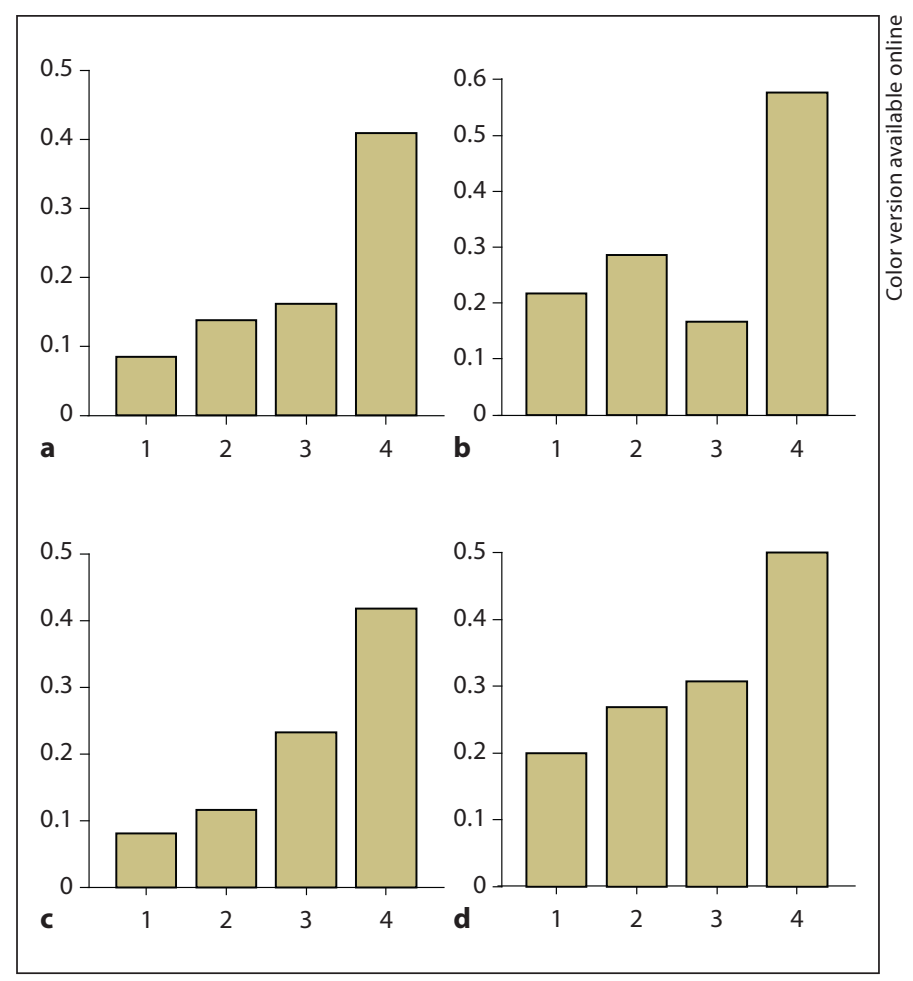

Fig. 1. Proportion of patients experiencing early mortality or mortality/disability at every quartile of EPI level and NE/DHPG ratio. a Mortality by EPI quartile. b Mortality/disability by EPI quartile. c Mortality by NE/DHPG quartile. d Mortality/disability by NE/DHPG quartile. Figures illustrate greatest mortality (a) and mortality/disability (b) rates in the highest quartile of EPI level, with approximately twice as many adverse outcomes within the 4 th quartile $\left(p=0.005\right.$ and $p=0.003$ for the $\chi^{2}$ linear association statistic). For the NE/DHPG ratio, an incremental increase in mortality (c) and mortality/disability (d) rates is observed at each subsequent quartile ( $\mathrm{p}=0.012$ and $\mathrm{p}=0.018$ for the $\chi^{2}$ test linear association statistic).

tality or disability was related to $\mathrm{H} / \mathrm{H}$ grade $(\mathrm{p}<0.001)$, NC $(\mathrm{p}=0.018)$, infarction $(\mathrm{p}<0.001)$ and elevated ICP $(\mathrm{p}=0.002)$.

The timing of CSF collection was comparable between the groups (table 3). All samples were successfully assayed, for a total of 612 measurements. In the univariate analysis, early mortality was positively associated with EPI levels $(p=0.002)$ and $N E / D H P G$ ratio $(p=0.003)$. Similarly, early mortality or disability was positively associated with EPI levels $(\mathrm{p}=0.004)$ and NE/DHPG ratio $(p=0.014)$. The proportion of patients experiencing early mortality or mortality/disability for each quartile of EPI level and NE/DHPG ratio is illustrated in figure 1, and the respective ROC curves are illustrated in figure 2. 
Table 1. Demographic, clinical, radiologic, and anatomical characteristics of the study groups

\begin{tabular}{|c|c|c|c|c|c|}
\hline & $\begin{array}{l}\text { Mortality } \\
(\mathrm{n}=21)\end{array}$ & $\begin{array}{l}\text { Disability } \\
(\mathrm{n}=12)\end{array}$ & $\begin{array}{l}\text { Mortality/ } \\
\text { disability }(\mathrm{n}=33)\end{array}$ & $\begin{array}{l}\text { No mortality/ } \\
\text { disability }(\mathrm{n}=69)\end{array}$ & $\mathrm{p}$ value \\
\hline \multicolumn{6}{|l|}{ Demographics } \\
\hline Age, years & $63 \pm 15$ & $56 \pm 18$ & $60 \pm 16$ & $56 \pm 13$ & $\mathbf{0 . 0 6 2 / 0 . 2 1 6}$ \\
\hline Female & $62(13)$ & $75(3)$ & $67(22)$ & $77(53)$ & $0.175 / 0.277$ \\
\hline Caucasian & $62(13)$ & $25(9)^{*}$ & $49(16)$ & $62(43)$ & $0.672 / 0.186$ \\
\hline \multicolumn{6}{|l|}{ Medical history } \\
\hline Hypertension & $71(15)$ & $75(9)$ & $73(24)$ & $57(39)$ & $0.306 / 0.115$ \\
\hline Tobacco use & $62(13)$ & $50(6)$ & $58(19)$ & $61(42)$ & $0.826 / 0.751$ \\
\hline EtOH use & 0 & $8(1)$ & $3(1)$ & $6(4)$ & $0.308 / 0.477$ \\
\hline Drug use & 0 & $8(1)$ & $3(1)$ & $3(2)$ & $0.497 / 0.695$ \\
\hline Cardiac disease & $19(4)$ & $8(1)$ & $15(5)$ & $9(6)$ & $0.163 / 0.255$ \\
\hline \multicolumn{6}{|l|}{ SAH aspects } \\
\hline $\mathrm{H} / \mathrm{H}$ grade $4 / 5$ & $91(19)$ & $75(9)^{* *}$ & $85(28)$ & $22(15)$ & $<0.001 /<0.001$ \\
\hline CT score $3 / 4$ & $79(15)$ & $67(8)$ & $74(23)$ & $55(38)$ & $0.075 / 0.070$ \\
\hline Anterior aneurysm location ${ }^{1}$ & $53(9)$ & $83(10)$ & $66(19)$ & $78(46)$ & $0.061 / 0.212$ \\
\hline Rehemorrhage & $5(1)$ & $17(2)$ & $9(3)$ & $4(3)$ & $0.641 / 0.297$ \\
\hline \multicolumn{6}{|l|}{ Complications } \\
\hline Neurogenic cardiomyopathy ${ }^{1}$ & $38(8)$ & $8(1)$ & $27(9)$ & $9(6)$ & $0.003 / 0.018$ \\
\hline Vasospasm 1 & $50(9)$ & $75(9)$ & $60(18)$ & $69(43)$ & $0.103 / 0.374$ \\
\hline Infarction & $52(11)$ & $50(6)^{* *}$ & $52(17)$ & $12(8)$ & $0.001 /<0.001$ \\
\hline Intracranial hypertension & $24(5)$ & $25(3)^{*}$ & $24(8)$ & $3(2)$ & $0.029 / 0.002$ \\
\hline \multicolumn{6}{|l|}{ Hospitalization } \\
\hline Duration, days & $7 \pm 3$ & $27 \pm 4^{* *}$ & $14 \pm 11$ & $20 \pm 5$ & $<0.001 / 0.004$ \\
\hline
\end{tabular}

For continuous variables, values represent means \pm SDs, and statistical calculations performed using the nonparametric rank-sum test. For categorical variables, values represent percentages (n shown in parentheses). 'Drug use' refers to sympathomimetic agents, and 'Cardiac disease' refers to history of coronary artery disease/myocardial infarction or preexisting structural myocardial dysfunction. 'Mortality/disability' was defined as a Glasgow Coma Scale score $\leq 10$ upon discharge from the neurological intensive care unit or adjoining intermediate care unit. Analyses performed include mortality versus no mortality, disability versus no mortality or disability, and mortality/disability versus no mortality/disability. $\mathrm{p}$ values are for $1 \mathrm{st} / 3 \mathrm{rd}$ analyses, whereas for $2 \mathrm{nd}$ analysis, ${ }^{*}$ indicates $\mathrm{p}<0.05$ and $^{* *}$ indicates $\mathrm{p}<0.01$.

${ }^{1}$ Angiogram was negative or not done in 14 cases, transthoracic echogram was uninterpretable in 2 cases (inadequate window), and no transcranial ultrasounds were recorded in 10 cases (inadequate windows).

Logistic regression (table 4) demonstrated positive associations between mortality and posterior aneurysm location ( $\mathrm{p}=0.034)$ and $\mathrm{NC}(\mathrm{p}=0.002)$, while age [OR 1.09 (95\% CI 1.01-1.17)], H/H grade [9.52 (1.19-77)], infarction [10.87 (1.22-100)], ICP elevation [32.26 (2-500)] and EPI [1.06 (1.01-1.10)] emerged as independent predictors, and DHPG emerged as a negative predictor [0.99 (0.99-1.00)]. For the outcome of mortality/disability, $\mathrm{NC}(\mathrm{p}=0.019)$, infarction ( $\mathrm{p}<0.001)$, and NE/DHPG $(\mathrm{p}=0.003)$ demonstrated positive associations, while $\mathrm{H} / \mathrm{H}$ grade [OR 21.74 (95\% CI 5.62-83.33)], ICP elevation [18.52 (1.93-166)] and EPI [1.05 (1.02-1.09)] emerged as independent predictors.

The predictive value of EPI was confirmed and the multivariate analysis validated by the bivariate analyses, and by alternative regression models constructed. Alternate models included each pertinent clinical variable indi- vidually entered along with EPI $(\mathrm{p}=0.001-0.028$ and $\mathrm{p}<0.001-0.004$ ), catecholamine variables DHPG and NE/ DHPG with EPI $(\mathrm{p}=0.003$ and $\mathrm{p}=0.002)$, and regression analyses with simultaneous entry of all variables $(\mathrm{p}=0.017$ and $p=0.007$ ), for the two outcome measures. In similarly constructed models, NE/DHPG predicted or tended to predict mortality/disability when entered with each individual pertinent clinical variable $(\mathrm{p}=0.006-0.077)$.

For the time-dependent outcome of mortality, Cox proportional (hazards) regression analysis (table 5) reinforced the association between mortality and age [HR 1.041 (95\% CI 1.003-1.080)], $\mathrm{H} / \mathrm{H}$ grade [6.90 (1.5431.25)], and EPI [1.032 (1.009-1.054)], and also revealed an association between NC and mortality [4.31 (1.5012.35)]. The corresponding survival curves comparing EPI quartile groups are presented in figure 3 . 
Fig. 2. ROC curves for early mortality according to EPI level and NE/DHPG ratio, and for EPI level and $\mathrm{H} / \mathrm{H}$ grade with a combined model. The figure illustrates the fair predictive value of EPI levels [AUC 0.72 (95\% CI 0.60-0.85, p = 0.002)], and of the NE/DHPG ratio [AUC 0.71 (95\% CI $0.55-0.84, p=0.003)$ ] for the outcome of early mortality. The figure also illustrates additive predictive value for early mortality when EPI levels and $\mathrm{H} / \mathrm{H}$ grade [AUC 0.81 ([95\% CI 0.70-0.92, p < 0.001)] are entered in combination [AUC 0.89 (95\% CI $0.82-0.95, \mathrm{p}<0.001)]$.

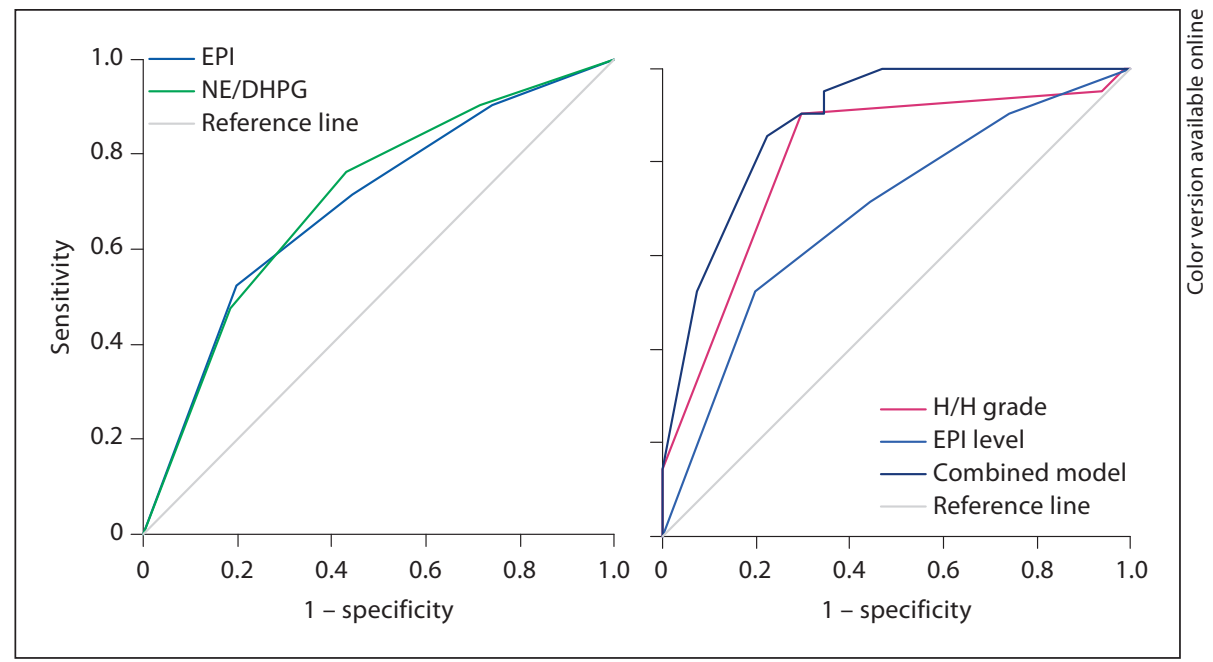

\section{Discussion}

In SAH, numerous biomarkers have been evaluated as potential predictors of outcome. Serum markers of inflammation [18], free-radical activity [19], coagulation function [20], cardiac injury [21] and neuronal injury [22]; CSF markers of inflammation [23], oxidation [24] and axonal damage [25]; cerebral microdialysate excitatory amino acid levels and markers of anaerobic metabolism [26]; urine albumin levels [27], and genetic profiles [28] have all been identified as possible outcome determinants following SAH. Complex interactions between different physiological systems may explain the diversity of biomarkers investigated as potential outcome predictors in SAH. Since intense sympathetic activation may initiate and participate in these various processes $[7,8]$, catecholamines may represent a valuable yet understudied group of biomarkers for predicting outcome.

Central sympathetic function is mediated by noradrenergic neurons in the locus ceruleus of the pons, and by the $A_{1}-A_{2}$ noradrenergic and $C_{1}-C_{3}$ adrenergic nuclear clusters in the ventrolateral medulla [29]. Studies have identified a prominent role for the $\mathrm{C}_{1}-\mathrm{C}_{3}$ nuclei in mediating sympathetic output for chemoreceptor and baroreceptor cardiovascular reflexes [29], for activation of sympathoneural defensive responses by release of adrenomedullary catecholamines [29], for controlling physiological responses during physical exertion and exercise [29], and in the development of hypertension [30]. Conversely, the neurons of the $\mathrm{A}_{1}-\mathrm{A}_{3}$ cluster activate the hypothalamic production and pituitary release of vasopressin and corticoliberin, and provide local inhibition to
Table 2. Final neurological condition and day of death or last clinical assessment

\begin{tabular}{lccc}
\hline & $\begin{array}{l}\text { Early } \\
\text { mortality }\end{array}$ & $\begin{array}{l}\text { Mortality/ } \\
\text { disability }\end{array}$ & $\begin{array}{l}\text { No mortality/ } \\
\text { disability }\end{array}$ \\
\hline $\begin{array}{l}\text { Number of patients } \\
\text { Day of death }\end{array}$ & 21 & 33 & 69 \\
$\begin{array}{l}\text { Day of final clinical } \\
\quad \text { examination }\end{array}$ & $7(4-10)$ & - & - \\
$\begin{array}{l}\text { Last GCS } \\
\text { (4) }\end{array}$ & $4(3-6)$ & $10(6-29)$ & $20(15-24)$ \\
\end{tabular}

Last GCS = Final Glasgow Coma Scale test performed on day of death or by day 30 . Values represent medians (IQR shown in parentheses).

${ }^{1}$ Combinations of factors contributed to patient demise, including severe initial clinical presentation, intracranial hypertension, cerebral edema, cardiac dysfunction, cerebral ischemia, and rehemorrhage. The single most proximate process responsible for death involved persisting coma leading to discontinuation of support in 9 patients, marked ICH in 5 patients, global or focal cerebral edema in 2 patients, multiple or large cerebral infarctions in 2 patients, myocardial infarction with heart failure in 1 patient, cardiac arrest in 1 patient, and rehemorrhage causing clinical worsening and decision to discontinue support in 1 patient.

the adjacent $\mathrm{C}_{1}-\mathrm{C}_{3}$ cells [29]. Via diffuse neocortical, limbic, thalamic and hypothalamic projections, the locus ceruleus primarily modulates global cerebral arousal, attention, vigilance, memory and behavior [29].

In the CNS, EPI and NE are metabolized to DHPG, given the presence of monoamine oxidase and the absence of catechol-O-methyltransferase [31]. NE/DHPG 
Table 3. Timing of sample collections and catecholamine levels for the study groups

\begin{tabular}{|c|c|c|c|c|c|c|}
\hline & \multicolumn{3}{|l|}{ Early mortality } & \multicolumn{3}{|c|}{ Mortality/disability } \\
\hline & mortality & no mortality & $\mathrm{p}$ value & $\begin{array}{l}\text { mortality/ } \\
\text { disability }\end{array}$ & $\begin{array}{l}\text { no mortality/ } \\
\text { disability }\end{array}$ & $\mathrm{p}$ value \\
\hline Onset to collection, $\mathrm{h}$ & $28 \pm 15$ & $28 \pm 13$ & 0.820 & $27 \pm 14$ & $28 \pm 14$ & 0.821 \\
\hline $\mathrm{EPI}, \mathrm{pg} / \mathrm{ml}$ & $28.61 \pm 26$ & $11.86 \pm 15.31$ & 0.002 & $26.75 \pm 27.38$ & $9.83 \pm 9.83$ & 0.004 \\
\hline $\mathrm{NE}, \mathrm{pg} / \mathrm{ml}$ & $168.04 \pm 176.97$ & $144.71 \pm 124.06$ & 0.548 & $179.40 \pm 204.00$ & $135.22 \pm 85.01$ & 0.966 \\
\hline DHPG, pg/ml & $482.47 \pm 367.02$ & $652.66 \pm 374.05$ & 0.063 & $518.51 \pm 350.47$ & $665.02 \pm 382.72$ & 0.080 \\
\hline DOPA, pg/ml & $1,024.5 \pm 505.8$ & $951.9 \pm 438.1$ & 0.378 & $1,027.8 \pm 432.9$ & $937.7 \pm 459.9$ & 0.108 \\
\hline Dopamine, $\mathrm{pg} / \mathrm{ml}$ & $132.46 \pm 200.67$ & $154.00 \pm 416.59$ & 0.611 & $146.31 \pm 262.12$ & $151.12 \pm 428.71$ & 0.362 \\
\hline DOPAC, $\mathrm{pg} / \mathrm{ml}$ & $12,880 \pm 8,625$ & $10,744 \pm 6,693$ & 0.356 & $12,632 \pm 7,829$ & $1,0491 \pm 6,735$ & 0.169 \\
\hline NE/DHPG & $0.425 \pm 0.273$ & $0.368 \pm 1.23$ & 0.003 & $0.688 \pm 1.91$ & $0.233 \pm 0.122$ & 0.014 \\
\hline
\end{tabular}

For continuous variables, values represent means \pm SDs. p values calculated using the nonparametric rank-sum test.

Table 4. Logistic regression analyses for predictors of early mortality and mortality/disability

\begin{tabular}{|c|c|c|c|c|c|c|c|c|}
\hline & \multicolumn{4}{|l|}{ Mortality } & \multicolumn{4}{|l|}{ Mortality/disability } \\
\hline & \multicolumn{2}{|l|}{ bivariate } & \multicolumn{2}{|l|}{ multivariate } & \multicolumn{2}{|l|}{ bivariate } & \multicolumn{2}{|l|}{ multivariate } \\
\hline & OR & $\mathrm{p}$ value & OR & $\mathrm{p}$ value & OR & $\mathrm{p}$ value & OR & $\mathrm{p}$ value \\
\hline Age & $1.04(1.00-1.08)$ & 0.051 & $1.09(1.01-1.17)$ & 0.024 & - & & - & \\
\hline $\mathrm{H} / \mathrm{H}$ grade & $23(4.88-100)$ & $<0.001$ & $9.52(1.19-77)$ & 0.034 & $20(6.62-62.5)$ & $<0.001$ & $21.74(5.62-83.33)$ & $<0.001$ \\
\hline CT score & $2.86(0.87-9.35)$ & 0.083 & NS & & $3.85(0.92-5.95)$ & 0.074 & NS & \\
\hline Anterior aneurysm & $0.30(0.09-0.91)$ & $<0.034$ & NS & & - & & - & \\
\hline NC & $6.41(1.98-20.83)$ & 0.002 & NS & & $3.88(1.25-12.05)$ & 0.019 & NS & \\
\hline Infarction & $5.26(1.88-14.71)$ & 0.002 & $10.87(1.22-100)$ & 0.033 & $8.13(2.97-22.22)$ & $<0.001$ & NS & \\
\hline ICP elevations & $4.74(1.23-18.52)$ & 0.024 & $32.26(2-500)$ & 0.015 & $10.75(2.13-52.63)$ & 0.004 & $18.52(1.93-166)$ & 0.011 \\
\hline EPI & $1.04(1.02-1.07)$ & 0.002 & $1.06(1.01-1.10)$ & 0.009 & $1.05(1.02-1.09)$ & 0.001 & $1.05(1.02-1.09)$ & 0.004 \\
\hline DHPG & $0.99(0.997-1.00)$ & 0.066 & $0.99(0.99-1.00)$ & 0.010 & $1.00(1.00-1.00)$ & 0.07 & NS & \\
\hline NE/DHPG & NS & & NS & & $49.38(3.69-661)$ & 0.003 & NS & \\
\hline
\end{tabular}

Multivariate logistic regression model for mortality incorporates age, $\mathrm{H} / \mathrm{H}$ grade, $\mathrm{CT}$ score, aneurysm location, NC, infarction, elevated ICP, and the variables EPI, DHPG, NE/DHPG. Multivariate model for mortality/disability incorporates hypertension, H/H grade, CT score, NC, infarction, elevated ICP, and the variables EPI, DHPG, NE/DHPG. Values in parentheses are 95\% CIs.

provides a relative measure of NE release to uptake, and serves as an index of active noradrenergic function [32]. In human subjects, plasma NE/DHPG progressively increases with increasing sympathetic stimulation [32], and CSF ratios increase with sympathetic disinhibition [33], while sympatholytics decrease the ratio in animal studies [34]. As a precursor in the synthesis of NE and EPI, DA represents an essential constituent for catecholaminergic compound production, and DA release increases in response to physiologic stress [35]. Similarly, it has been proposed that CSF levels of DOPA may reflect central catecholamine biosynthetic capacity [33], and that CSF
DOPAC levels may provide an estimate of overall central catecholamine transmission [36].

The results of our study demonstrate that CSF EPI levels independently predicted which patients died or were disabled from SAH, with a 3-6\% increased risk for each picogram per milliliter increase in EPI. Results also indicate that EPI levels may contribute additively to $\mathrm{H} / \mathrm{H}$ grade in a combined model for predicting early mortality. Our findings are consistent with those of a prior report, which identified elevated EPI and NE levels in the plasma and CSF of SAH patients with adverse outcomes [37]. This prior investigation was, however, limited by very 
Table 5. Proportional-hazards analysis for the time-dependent outcome of mortality

\begin{tabular}{lcl}
\hline & HR & p value \\
\hline Age & $1.041(1.003-1.080)$ & $\mathbf{0 . 0 3 4}$ \\
H/H grade & $6.90(1.54-31.25)$ & $\mathbf{0 . 0 1 2}$ \\
CT score & $1.50(0.368-6.10)$ & 0.573 \\
Anterior aneurysm & $0.662(0.228-1.92)$ & 0.448 \\
NC & $4.31(1.50-12.35)$ & $\mathbf{0 . 0 0 7}$ \\
Infarction & $2.47(0.764-8.0)$ & 0.131 \\
ICP elevation & $2.61(0.574-11.90)$ & 0.215 \\
EPI & $1.032(1.009-1.054)$ & $\mathbf{0 . 0 0 5}$ \\
\hline
\end{tabular}

$\mathrm{HR}=$ Hazard ratio. Regression model incorporates variables age, $\mathrm{H} / \mathrm{H}$ grade, $\mathrm{CT}$ score, aneurysm location, $\mathrm{NC}$, infarction, elevated ICP, and EPI. Figures in parentheses are 95\% CIs.

small patient numbers and a lack of rigorous statistical analysis [37]. Critics of that study also questioned whether the presence of EPI in the CSF may have been due to entry from the systemic circulation at the time of hemorrhage [38]. However, CSF EPI has also been detected and quantified in various (nonhemorrhagic) psychiatric [39], neurodegenerative [40], and pain-related conditions [41], and has been found to be increased in hyperactive states, such as anxiety or psychosis [42, 43], and decreased in despondent states including depression [44, 45].

The findings of our investigation are consistent with evidence in the literature linking sympathetic function with diverse systemic and multiorgan derangements, including hypertension [46], myocardial ischemia [47], congestive heart failure [48], cardiac tachyarrhythmias [49], sudden cardiac mortality [50], abnormal renal natriuresis [51], enhanced platelet aggregation [52] and impaired immune function [53]. Sympathetically mediated neurogenic cardiopulmonary complications have been related to adverse outcomes following SAH [54], and sympathetic activity has been connected to increased inflammation and enhanced thrombosis $[7,8]$, the markers of which predict detrimental outcomes in patients with SAH $[18,20]$. Adrenergic activity has also been invoked in the production of cerebral vasospasm [55], although as in our study infarction may also occur independent of any spasm [56], as well as in the development of cerebral edema following ICH [57]. Thus, elevated catecholamine levels may induce a state of generalized multiorgan dysfunction, which promotes deleterious clinical outcomes beyond simply the extent of neurological impairment.

CSF Catecholamines and SAH Outcome

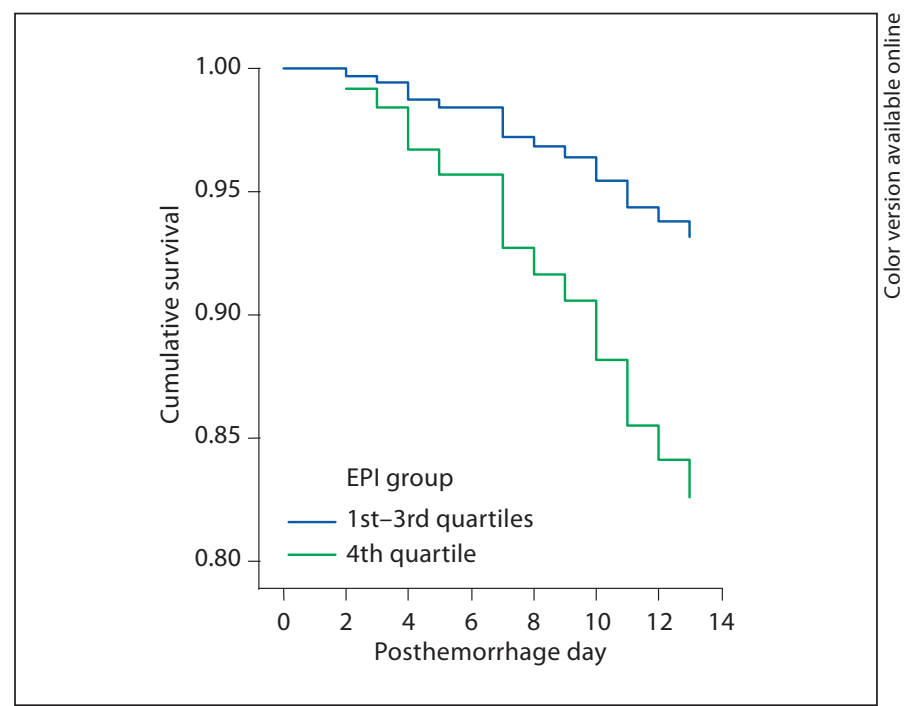

Fig. 3. Proportional hazards analysis survival curve. Adjusted cumulative survival by posthemorrhage day for patients in the highest (4th) quartile of EPI level versus those in the lower (1st-3rd) EPI quartiles. The figure illustrates consistently greater mortality rates among the 4th-quartile group over time [HR 2.72 (95\% CI $1.01-7.30, \mathrm{p}=0.048)]$.

The findings of our research contribute to the prognostic armamentarium available for assessing outcome in patients with severe-grade SAH. However, our results may also serve to optimize subject selection for future sympatholytic trials, and thereby provide a new avenue for novel therapeutic strategies and interventions. Catecholamine receptor blockade has been proven advantageous in various hyperadrenergic cardiovascular states [46-49], and may also potentially improve outcomes in neurogenic cardiopulmonary processes [58]. As such, clinical biomarkers need not be used solely for prognostic information (or improperly employed to promote clinical nihilism), but instead may be utilized to advance medical research and present novel treatment opportunities.

Limitations to our study include specimen contamination by plasma, which may theoretically confound the measurement of central catecholamine analytes. However, SAH by nature involves hemorrhage into the CSF, making this an unavoidable consequence. The ineligibility of devastated SAH patients whose condition was immediately terminal or in whom support was discontinued early, thereby precluding ventriculostomy, may have excluded those patients with the greatest elevations in central catecholamine levels. Thus, our results may have underestimated the true differences in central catechol- 
aminergic profiles between patients with and without adverse outcomes. The variable use of different anesthetic, sedating and analgesic agents at various doses and durations, and the absence of a standard continuous measure of intensity and duration of action, as well as the unquantifiable impact upon the sympathetic output in any given individual, may represent an additional drawback. A final limitation is the small size of our study in that larger subject numbers may have provided greater power and fidelity in identifying differences between the groups. Nevertheless, the narrow CIs for EPI indicate the precision of our ORs and results.

Positive aspects of our study include the consistency of our results with the existing literature regarding determinants of mortality and disability, including age $[59,60]$, severe clinical grade $[59,60]$, presence of neurogenic cardiomyopathy [9], cerebral infarction $[59,60]$, and ICP elevations [61]. Additional attributes include the plausibility of our findings, given the proven activation of the sympathetic nervous system following $\mathrm{SAH}$ [6], the established connection between sympathetic activity and systemic inflammation and thrombosis $[7,8]$, and the association between markers of inflammation and thrombosis with outcome $[18,20]$. Our findings are also internally consistent, revealing elevations in several catecholaminergic parameters among patients with adverse outcomes. Finally, the absence of a contemporary study evaluating CSF catecholamine as potential biomarkers indicates the unique nature of our study.

\section{Conclusion}

In conclusion, $\mathrm{SAH}$ patients who die in hospital or who are disabled by 30 days demonstrate greater central activation of the sympathetic nervous system than those who survive without severe impairment. CSF catecholamine levels and ratios may serve as early indicators of clinical neurological outcome, and therefore may serve as valuable biomarkers in the prognostication of patients with SAH. Additional studies are needed to verify these findings.

\section{Acknowledgments}

The authors express gratitude to the nurses of the neurological intensive care unit from the Jefferson Hospital of Neurosciences at Thomas Jefferson University Medical Center for their assistance in this project. This project represents a collaboration between the Neurocritical Care Division in the Department of Neurology at Thomas Jefferson Medical Center, and the Section on Neuroendocrinology of the Reproductive and Adult Endocrinology Program at the National Institutes of Health. This research study was supported in part by the Intramural Research Program of the NIH/NICHD.

\section{Disclosure Statement}

The authors have no financial affiliations or conflicts of interest to announce.

\section{References}

1 Castellanos M, Serena J: Applicability of biomarkers in ischemic stroke. Cerebrovasc Dis 2007;24:7-15.

-2 Foerch C, Montaner J, Furie K, et al: Searching for oracles? Blood biomarkers in acute stroke. Neurology 2009;73:393-399.

3 Whiteley W, Wardlaw J, Dennis M, et al: Blood biomarkers for the diagnosis of acute cerebrovascular diseases - a prospective cohort study. Cerebrovasc Dis 2011;32:141-147.

-4 Hemphill J, Bonovich D, Besmertis L, et al: The ICH Score - A simple reliable grading scale for intracerebral hemorrhage. Stroke 2001;32:891-897.

5 Ruiz-Sandoval J, Chiquete E, Romero-Vargas $\mathrm{S}$, et al: Grading scale for prediction of outcome in primary intracerebral hemorrhages. Stroke 2007;38:1641-1644.

-6 Naredi S, Lambert G, Eden E, et al: Increased sympathetic nervous activity in patients with nontraumatic subarachnoid hemorrhage. Stroke 2000;31:901-906.
7 Gao C, Liu X, Shi H, et al: Relationship between sympathetic nervous system activity and inflammatory response after subarachnoid hemorrhage in a perforating canine model. Auton Neurosci 2009;147:7074 .

-8 Larsson P, Wallen N, Hjemdahl P: Norepinephrine-induced human platelet activation in vivo is only partly counteracted by aspirin. Circulation 1994;89:1951-1957.

-9 Lee V, Oh J, Mulvagh S, et al: Mechanisms in neurogenic stress cardiomyopathy after aneurysmal subarachnoid hemorrhage. Neurocrit Care 2006;5:243-249.

10 Welsh P, Barber M, Langhorne P, et al: Associations of inflammatory and haemostatic biomarkers with poor outcome in acute ischaemic stroke. Cerebrovasc Dis 2009;27: 247-253.

11 Kerr G, Gautamananda R, Wu O, et al: Elevated troponin after stroke - a systematic review. Cerebrovasc Dis 2009;28:220-226.
12 Julien J, Bandeen-Roche K, Tamargo R: Validation of an aneurysmal subarachnoid hemorrhage grading scale in 1,532 consecutive patients. Neursurgery 2008;63:204-211.

13 Eisenhofer G, Goldstein D, Stull R, et al: Simultaneous liquid chromatographic determination of 3,4-dihydroxyphenylglycol, catecholamines, and 3,4-dihydroxyphenylalanine in plasma, and their responses to inhibition of monoamine oxidase. Clin Chem 1986;32:2030-2033.

14 Kramer A, Hehir M, Nathan B, et al: A comparison of 3 radiographic scales for the prediction of delayed ischemia and prognosis following subarachnoid hemorrhage. J Neurosurg 2008;109:199-207.

15 Banki N, Kopelnik A, Tung P, et al: Prospective analysis of prevalence, distribution, and rate of recovery of left ventricular systolic dysfunction in patients with subarachnoid hemorrhage. J Neurosurg 2006; 105:15-20. 
16 Alexandrov A, Neumyer M: Diagnostic criteria for cerebrovascular ultrasound; in Alexandrov A (ed): Cerebrovascular Ultrasound in Stroke Prevention and Treatment. New York, Blackwell, 2004, pp 81-129.

17 Wozniak M, Sloan M, Rothman M, et al: Detection of vasospasm by transcranial Doppler sonography - the challenges of the anterior and posterior cerebral arteries. J Neuroimaging 1996;6:87-93.

18 Mack W, Mocco J, Hoh D, et al: Outcome prediction with serum intracellular adhesion molecule 1 levels after aneurysmal subarachnoid hemorrhage. J Neurosurg 2002; 96:71-75.

-19 Polidori M, Frei B, Rordorf G, et al: Increased levels of plasma cholesteryl ester hydroperoxides in patients with subarachnoid hemorrhage. Free Radic Biol Med 1997;23:762-767.

-20 Morga R, Czepko R, Dembińska-Kieć A, Danilewicz B: Assessment of the haemostatic system in patients surgically treated for ruptured cerebral aneurysm. Neurol Neurochir Pol 2007;41:296-305.

-21 Ramappa P, Thatai D, Coplin W, et al: Cardiac troponin I - a predictor of prognosis in subarachnoid hemorrhage. Neurocrit Care 2008;8:398-403.

-22 Stranjalis G, Korfias S, Psachoulia C, et al: The prognoistic value of serum S-100 protein in spontaneous subarachnoid hemorrhage. Acta Neurochir 2007;149:231-237.

$\checkmark 23$ Kaynar M, Tanriverdi T, Kafadar A, et al: Detection of soluble intracellular adhesion molecule 1 and vascular cell adhesion molecule 1 in both cerebrospinal fluid and serum of patients after aneurysmal subarachnoid hemorrhage. J Neurosurg 2004;101:10301036.

24 Lin C, Hsu Y, Lin T, et al: increased levels of F2 isoprostanes following aneurysmal subarachnoid hemorrhage in humans. Free Radic Biol Med 2006;40:1466-1473.

25 Petzold A, Keir G, Kay A, et al: Axonal damage and outcome in subarachnoid hemorrhage. J Neurol Neurosurg Psychiatry 2006; 77:753-759.

-26 Staub F, Graf R, Gabel P, et al: Multipe interstitial substances measured by microdialysis in patients with subarachnoid hemorrhage. Neursurgery 2000;47:1106-1115.

-27 Terao Y, Takada M, Tanabe T, et al: Microalbuminemia is a prognostic predictor in aneurysmal subarachnoid hemorrhage. Intensive Care Med 2007;33:1000-1006.

28 Lanterna L, Ruigrok Y, Alexander S, et al: Meta-analysis of APO-E genotype and subarachnoid hemorrhage. Neurology 2007;69: 766-775.

29 Vinken P, Bruyn G (eds): The Autonomic Nervous System. Amsterdam, Elsevier, 1999.

30 Chalmers J, Howe P, Costa M, et al: Adrenaline synthesizing nerve cells in the medulla of normotensive and hypertensive rats. Clin Exp Pharmacol Physiol 1981;8:459-462.
31 Eisenhofer G, Kopin I, Goldstein D: Catecholamine metabolism: a contemporary view with implications for physiology and medicine. Pharmacol Rev 2004;56:331-349.

32 Lenders J, Willemsen J, Beissel T, et al: Value of the plasma norepinephrine/3,4-dihydroxyphenylglycol ratio for the diagnosis of pheochromocytoma. Am J Med 1992;92: 147-152.

33 Raskind M, Peskind E, Holmes C, et al: Patterns of cerebrospinal fluid catechols support increased central noradrenergic responsiveness in aging and Alzheimer's disease. Biol Psychiatry 1999;46:756-765.

- 34 Du X, Esler M, Dart A: Sympatholytic action of intravenous amiodarone in the rat heart. Circulation 1995;91:462-470.

35 Feenstra M: Dopamine and noradrenaline release in the prefrontal cortex in relation to unconditioned and conditioned stress and reward. Prog Brain Res 2000;126:133-163.

36 Ebinger G: The significance of homovanillic acid and 3,4-dihydroxyphenylacetic acid concentrations in human lumbar cerebrospinal fluid. J Neurochem 1987;48:17251729.

- 37 Dilraj A, Botha J, Rambiritch V, et al: Levels of catecholamines in plasma and cerebrospinal fluid in aneurysmal subarachnoid hemorrhage. Neurosurgery 1992;31:42-51.

38 Wood J: Comment on: Levels of catecholamine in plasma and cerebrospinal fluid in aneurysmal subarachnoid hemorrhage. Neurosurgery 1992;31:51-52.

39 Berger P, King R, Lemoine P, et al: Cerebrospinal fluid epinephrine concentrations discrimination of subtypes of depression and schizophrenia. Psychopharm Bull 1984; 20:412-415

40 Chia L, Cheng F, Kuo J: Monoamines and their metabolites in plasma and lumbar cerebrospinal fluid of Chinese patients with Parkinson's disease. J Neurol Sci 1993;116:125134.

41 Strittmatter M, Grauer M, Isenberg E, et al: Cerebrospinal fluid neuropeptides and monoaminergic transmitters in patients with trigeminal neuralgia. Headache 1997; 37:211-216.

42 Gjerris A, Rafaelsen O, Christensen N: CSFadrenaline in somatizing depression. Acta Psychiatr Scand 1987;75:516-520.

43 Gattaz W, Gasser T, Beckmann H: Multidimensional analysis of the concentrations of 17 substances in the csf of schizophrenics and controls. Biol Psychiatry 1985;20:360366.

44 Christensen N, Vestergaard P, Sorensen T, et al: Cerebrospinal fluid adrenaline and noradrenaline in depressed patients. Acta Psychiatr Scand 1980;61:178-182.

45 Gjerris A: Do concentrations of neurotransmitters in lumbar CSF reflect cerebral dysfunction in depression? Acta Psychiatr Scand 1988;345:21-24.
46 Fisher J, Paton J: The sympathetic nervous system and blood pressure in humans. J Hum Hypertens 2011.

47 Remme W: The sympathetic nervous system and ischemic heart disease. Eur Heart J 1998; 19:62-71.

48 Esler M, Kaye D, Lambert G, et al: Adrenergic nervous system in heart failure. Am J Cardiol 1997;80:7-14.

49 Volders P: Novel insights into the role of the sympathetic nervous system in cardiac arrhythmogenesis. Heart Rhythm 2010;7: 1900-1906.

50 Dhalla N, Adameova A, Kaur M: Role of catecholamine oxidation in sudden cardiac death. Fundam Clin Pharmacol 2010;24: 539-546.

-51 Audibert G, Steinmann G, De Talance N, et al: Endocrine response after severe subarachnoid hemorrhage related to sodium and blood volume regulation. Anesth Analg 2009;108:1922-1928.

52 Petidis K, Douma S, Doumas M, et al: The interaction of vasoactive substances during exercise modulates platelet aggregation in hypertension and coronary artery disease. BMC Cardiovasc Disord 2008;27:11.

53 Emsley H, Hopkins S: Post-stroke immunodepression and infection - an emerging concept. Infect Disord Drug Targets 2010;10:91-97.

54 Bruder N, Rabinstein A: Cardiovascular and pulmonary complications of aneurysmal subarachnoid hemorrhage. Neurocrit Care 2011.

55 Lobato R, Marin J, Salaices M, et al: Effect of expermental subarachnoid hemorrhage on the adrenergic innervation of cerebral arteries. J Neurosurg 1980;53:477-479.

56 Vergouwen M, Ilodigwe D, Macdonald L: Cerebral Infarction after subarachnoid hemorrhage contributes to poor outcome by vasospasm dependent and independent effects. Stroke 2011;42:924-929.

-57 Sansing L, Messe S, Cucchiara B, et al: Antiadrenergic medications and edema development after intracerebral hemorrhage. Neurocrit Care 2011;14:395-400

58 Cruickshank J, Neil-Dwyer G, Degaute J, et al: Reduction of stress/catecholamine induced cardiac necrosis by beta-1 selective blockade. Lancet 1987;2:585-589.

-59 Rosengart A, Schultheiss K, Tolentino J, et al: Prognostic factors for outcome in patients with aneurysmal subarachnoid hemorrhage. Stroke 2007;38:2315-2321.

-60 Shirao S, Yoneda H, Kunitsugu I, et al: Preoperative prediction of outcome in 283 poorgrade patients with subarachnoid hemorrhage - a project of the Japan Neurosurgical Society. Cerebrovasc Dis 2010;30:105-113.

-61 Heuer G, Smith M, Elliott J, et al: Relationship between intracranial pressure and other clinical variables in patients with aneurysmal subarachnoid hemorrhage. J Neurosurg 2004;101:408-416. 